\title{
Distributed Control and Power Management Strategy for an Autonomous Hybrid Microgrid with Multiple Sub- Microgrids
}

\author{
Mohammad Abuhilaleh, Li Li, Jianguo Zhu \\ School of Electrical and Data Engineering \\ University of Technology Sydney \\ Sydney, NSW 2007, Australia \\ Mohammad.m.abuhilaleh@student.uts.edu.au,Li.Li@uts.edu.au, \\ Jianguo.Zhu@uts.edu.au
}

\begin{abstract}
This paper proposes a novel approach of distributed coordination control for multiple sub-microgrids (SMGs) within a hybrid AC/DC microgrid. The conventional control approach for managing power flow among AC and DC SMGs is based on the proportional power sharing principle. This is mainly implemented by equalising the normalized voltage at the DC side and the frequency at the AC side for any interfaced SMGs. The proposed method suggests a distributed control system that ensures a total controllability for the interlinking converters. It overcomes the total dependency on a specific variable for power exchange. The proposed method not only enables control of the power flow between SMGs but also ensures the continuity of power transfer if any single SMG fails. Three case studies are presented to demonstrate the validity and capability of the proposed approach using the MATLAB/Simulink software. From the obtained results, it is found that the proposed control system provides a high level of flexibility in managing the power flow among SMGs.
\end{abstract}

Index Terms - Hybrid microgrid, droop method, distributed control

\section{INTRODUCTION}

Microgrid is gaining momentum in the power system research field due to its economic, environmental, and sustainable incentives. Moreover, such a system is considered to be the solution for the complexity of integrating diverse renewable energy technologies. Distributed generators (DGs), storage elements (SEs) and loads are considered to be the main components in microgrids [1]. The cooperation of these elements under suitable control system supervision can ensure a seamless and secure operation of such system. DGs can be segmented into renewable energy sources (photovoltaic, wind, and wave energy sources) and non-renewable ones (nuclear, diesel and coal-powered systems). The SEs have a wide range of classifications according to the capacity and charging specifications. Ultra-capacitors, batteries, and flywheels are the most common SEs used in the modern electrical systems. There are three main structures of microgrids: AC, DC, and hybrid AC/DC microgrid. These structures are categorised

\author{
M.J. Hossain \\ School of Engineering, \\ Macquarie University, \\ North Ryde NSW 2109, Australia \\ jahangir.hossain@mq.edu.au
}

based on how the DGs of the DC and AC microgrids are connected to the loads [2-4].

AC microgrids have been the focus of research in the recent decade due to the advantages of using clean and sustainable energy sources. They also have the ability to integrate the renewable energy sources with non-renewable ones, all within the main grid and without the need for power conversion [5-7]. On the other hand, there is an increased interest in DC microgrids, not only to suit lots of renewable energy sources that naturally generate power in the DC form (photovoltaic and fuel cell), but also to meet the growing penetration of modern DC loads. This interest in the DC and AC microgrids is promoting to link these systems together by a bidirectional power converter and form the hybrid microgrid (HMG). Such a system is strongly recommended to overcome the power shortage in any part of the hybrid system. Most of the literature proposes a HMG structure that consists of a single interface AC and DC microgrid. However, the trend for linking multiple microgrids with different voltage and frequency levels is gaining an increased interest in the power system industry field. This is due to the many benefits of such arrangement [8].

The microgrid can work in two operation modes: the gridconnected mode where the voltage and frequency are regulated by the main grid, and the autonomous mode which is a more realistic situation in rural areas. The main challenge in the autonomous arrangement is the power management and control coordination [9]. This can be achieved by the local controller in every sub-microgrid (SMG) regulating the voltage and frequency at the AC bus and the voltage at the DC bus. These controllers are also responsible for managing the power flow within the SMGs. On the other hand, the transferable power from one SMG to another is controlled through the bidirectional interlinking converters (ILCs) which play a critical role in increasing the stability and reliability of the system $[10,11]$.

Conventionally, the droop control technique is widely used as a decentralized control method of the three microgrid structures mentioned before. In the case of the HMG which is our focus in this paper, many researches use the droop 
characteristic as indicators (representatives) of the power conditions in AC and DC SMGs [12-15]. This implies that the frequency and voltage should slightly vary when the active power changes at the AC and DC side. Depending on this variation, a sequential process is used so that the results of such processes are used as references to the ILCs. Thus, every individual SMG cooperates with other SMGs and shares the active power proportionally to its total capacity.

In the case of hybrid microgrid with multiple SMGs, the droop technique is used for every interfaced SMG. Thus, the $\mathrm{AC}$ bus works as a common bus and mediates the power flow from one SMG to another according to the SMG power condition. The frequency works as a transferable indicator between the SMGs even when the power flows from DC to DC SMG. Although this method obtains a high level of flexibility and reliability, it has many drawbacks that limit its implementation. For instance, the conventional droop method is not suitable when the hybrid microgrid consists of multiple SMGs. Moreover, once the AC SMG is disconnected from the hybrid microgrid, there will no longer be any power transfer in the system as the frequency of the AC SMG is what triggers the ILCs.

This paper proposes a new approach to the control strategy to manage the power flow among SMGs. A global control technique is implemented through the participation of the normalized variables of all SMGs to control the power flow through each ILC without relying on one single variable for each ILC. The organization for the rest of this paper is as follows. Section II illustrates the hybrid microgrid structure used in this work. Section III discusses the conventional control method and its advantages and disadvantages. Section IV presents the proposed control approach. Section V includes the case studies and simulation results that clarify the performance of the proposed control system. Finally, the conclusions are presented in Section VI.

\section{AUTONOMOUS HYBRID MICROGRID STRUCTURE}

Fig. 1 shows the schematic diagram of the hybrid AC/DC microgrids with multiple SMGs. In the AC SMG, every DG is emulated by a DC/AC converter that is linked to the local $\mathrm{AC}$ bus and directly connected to the common AC bus. The DC SMGs are linked to their local DC buses via voltage source inverters (VSIs), and each DC SMG is linked to the common $\mathrm{AC}$ bus by an ILC. The AC common bus links all the SMGs and mediates the power exchange that may occur. Each ILC acts as a bi-directional converter and operates in two modes depending on the direction of the power flow. When the HMG works in the grid connected mode, the intelligent transfer switch or the circuit breaker remains closed in the normal state. The switch opens, and the HMG operates in the autonomous mode if there is any planned (for example switch maintenances) or unplanned opening (such as emergency breakdowns). Adopting such a structure would boost the system efficiency through reduction of the conversion stages. Also, the power sharing in this structure increases the total power rating of the hybrid microgrid and improves the system reliability.

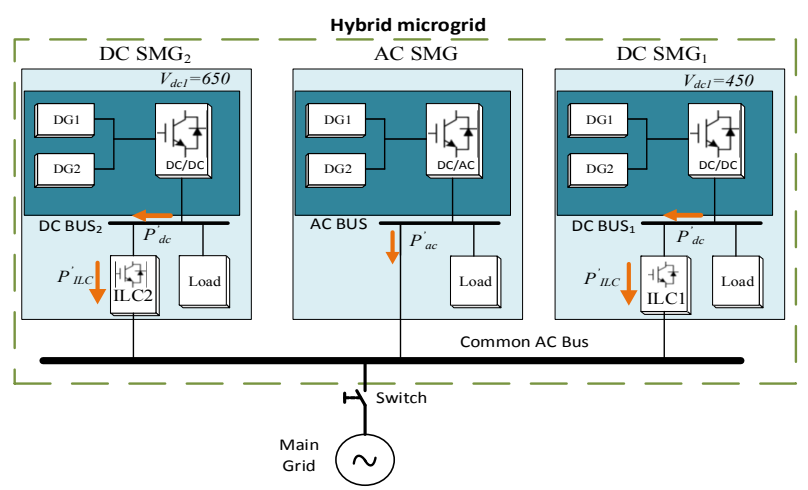

Figure 1. HMG structure with multiple SMGs

\section{Conventional Control Methodology}

According to the conventional control scheme used in power systems, droop control method is utilized in the primary control level for $\mathrm{AC}$ and $\mathrm{DC}$ SMGs and $\mathrm{AC} / \mathrm{DC} \mathrm{HMG}$ as follows:

\section{A. Droop control of the AC SMG}

In the AC SMG, the main aim of control system is to regulate the voltage and frequency at the $\mathrm{AC}$ bus, as well as managing the power flow and ensuring sufficiently accurate power sharing among the DGs. This can be obtained by using $(P-f)$ and $(Q-V)$ droop control loops. The basic concept of this method is to mimic the behaviour of synchronous generators. These loops are adapted to ensure an inductive output impedance and connect parallel inverters without using any physical communication links. The $P / f$ and $Q / V$ curves (Fig. 2) define the characteristic equations of the amplitude $\left(V_{i}\right)$ and frequency $\left(f_{i}\right)$ of the output voltage for the $i^{t h} \mathrm{DG}\left(\mathrm{DG}_{\mathrm{i}}\right)$ as the following:

$$
f_{i}=f_{\max }-\alpha_{i} \cdot P_{a c, i} ; \quad V_{i}=V_{\max }-\beta_{i} \cdot Q_{a c, i}
$$

where $V_{\max }$ and $f_{\max }$ are the maximum voltage magnitude and frequency at the no load condition. $P_{a c, i}$ and $Q_{a c, i}$ are the calculated output active and reactive power respectively. $\alpha_{i}$ and $\beta_{i}$ are the droop control gain factors which are usually tuned carefully after taking into account the maximum and minimum allowable voltage and frequency deviations $\left(V_{\max }\right.$, $f_{\max }, V_{\min }, \& f_{\min }$ ) and they are given as:

$$
\alpha_{i}=\frac{f_{\max }-f_{\min }}{P_{i, \text { max }}} ; \beta_{i}=\frac{V_{\text {max }}-V_{\text {min }}}{Q_{i, \text { max }}}
$$

\section{B. Droop control of the DC SMG}

In the DC SMG, the control system is almost similar to the $\mathrm{AC}$ one except that it only involves the active power. The $P / V$
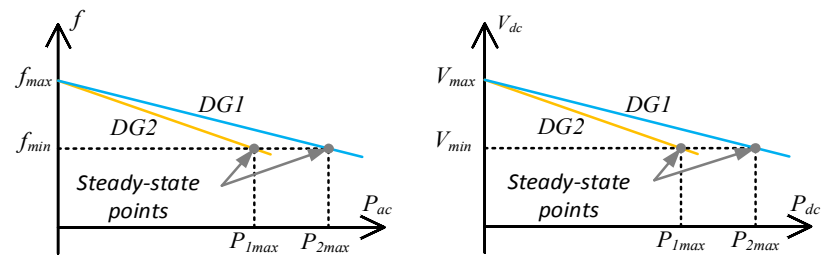

Figure 2. Active power droop characteristics of AC and DC SMGs 
droop control is also adopted to regulate the DC voltage that slightly varies with the active output power. Virtual output impedances loop is also utilized together with the droop loop to avoid the circulation current and ensure an inductive output impedance of the converter. The mathematical form that represents the $P / V$ droop control is given as:

$$
V_{d c, i}=V_{d c}^{\max }-\lambda_{i} \cdot P_{d c, i}
$$

where $V^{\max } d c$ is the maximum DC voltage at the no load condition. $\lambda_{i}$ is the droop control gain factor which reflects the rate of deviation in the output voltage $\left(V_{d c, i}\right) ; \lambda_{i}$ should be tuned carefully as a slight increase of the droop gain factor may result in a wide voltage range, which might not be accepted for some applications. However, such an increase in the voltage range yields more accurate power sharing. The droop gain is given by:

$$
\lambda_{i}=\frac{V_{d c, i}{ }^{\mathrm{max}}-V_{d c, i}^{\mathrm{min}}}{P_{d c, i}^{\max }}
$$

\section{Droop control of the $H M G$}

Once the voltage and frequency have been regulated in accordance with the power management strategy in each single SMG, the ILCs energise in the system and link each DC SMG with the common AC bus, not only to manage the transferable power among SMGs, but also to increase the whole system stability by reducing the voltage and frequency variations in each SMG. The conventional method for managing the power flow in HMG basically depends on the characteristics of the droop curves $(P / f$ in $\mathrm{AC} \mathrm{SMG}$ and $P / V$ in DC SMGs). That is because these curves reflect the amount of active power in each SMG. This is further shown through the merge of all the individual droop curves of all DGs into one curve for each SMG. The new droop curves with output frequency $f_{S}$ at the $\mathrm{AC}$ SMG and the voltage $V_{S, i}$ at the DC $\mathrm{SMG}_{i}$ can be given as follows:

$$
\begin{gathered}
f_{S}=f^{*}-\alpha_{S} P_{a c, S}, \quad \alpha_{S}=\frac{1}{\sum_{i=1}^{n} \frac{1}{a_{i}}} \\
V_{S, i}=V^{*}-\lambda_{S, i} P_{d c, S, i}, \quad \lambda_{S, i}=\frac{1}{\sum_{i=1}^{n} \frac{1}{\lambda_{i}}}
\end{gathered}
$$

where $\alpha_{S}$ and $\lambda_{S, i}$ are the combined droop gain of the total output active power $\left(P_{a c, S}\right.$ and $\left.P_{d c, S, i}\right)$ of the AC SMG and the $i^{\text {th }} \mathrm{DC}$ SMG (DC $\mathrm{SMG}_{i}$ ) respectively. Then, these droop curves undergo a normalization process so that it could be compared to each other as a standardized indicator of the power condition. The following expression represents the normalized frequency $\left(f_{S M G}^{\prime}\right)$ at AC SMG and voltage $\left(\dot{V}_{S M G, i}\right)$ at $\mathrm{DC} \mathrm{SMG}_{i}$

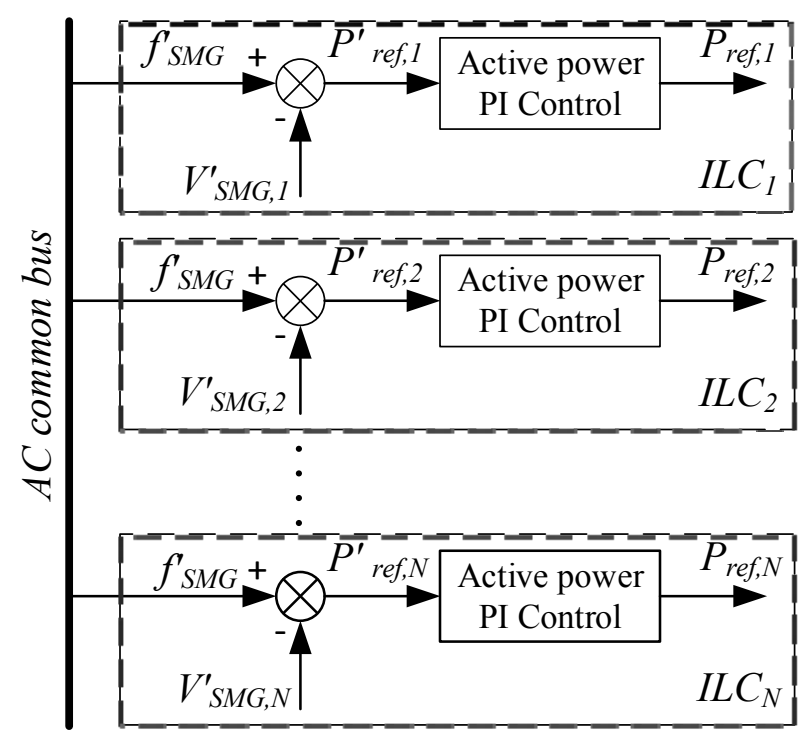

Figure 3. ILCs Conventional control method.

$$
\begin{aligned}
& f_{S M G}^{\prime}=\left(f_{S}-f_{\min }\right) /\left(f_{\max }-f_{\min }\right) \\
& V_{S M G, i}^{\prime}=\left(V_{S}-V_{\min }\right) /\left(V_{\max }-V_{\text {min }}\right)
\end{aligned}
$$

Now the normalized parameters are comparable since they have the same range scale which varies between 0 to 1 . According to this range, when the SMG has the maximum output power, the normalized value will be 0 and consequently the output voltage will be the minimum allowable value.

Once the normalized parameters $\left(f_{S M G}^{\prime}, \dot{V}_{S M G i}\right)$ are equalized, these parameters are simultaneously utilized as an input of the power management strategy, and the interfaced SMGs share an active power proportional to their ratings. Thus, the ILC manages the power flow between the involved SMGs and at the same time maintains the stability of the AC and DC SMGs. The per unit transferred power can be achieved by merging $f_{S M G}^{\prime}$ and $\dot{V}_{S M G i}$ obtained from (6) and can be given as:

$$
P_{r e f, i}^{\prime}=f_{S M G}^{\prime}-V_{S M G_{i}}^{\prime}
$$

A similar approach can be adopted for the HMG with a multiple SMGs structure. The difference here is that the normalized frequency is compared with each normalized voltage as shown in Fig. 3 and each DC SMG will deliver or absorb power to/from the AC bus based on $\dot{P}_{\text {ref }, i}$. The conventional control approach presented above can achieve an accurate power sharing among SMGs without any communication links among DGs or ILCs. On the other hand, the problem in such approach is that it can easily fail if the AC SMG disconnects for a planned (maintenance process) or unplanned condition (faults). In such case, the power transfer will no longer take place.

\section{PROPOSED CONTROL METHODOLOGY}


In order to avoid the interruption of power transfer among SMGs due to the isolation of the AC SMG from the whole system, a distributed control system approach is proposed in this work. The main idea is to implement an outer control loop (primary loop) as a local controller that includes the normalized factors $\left(f_{S M G}, \dot{V}_{S M G, 1}, \dot{V}_{S M G, 2}, \ldots, \dot{V}_{S M G, N}\right)$ of all SMGs in each ILC. Fig. 4 shows the total schematic diagram of the proposed distributed control system of ILCs.

In the proposed control strategy, the controller of each ILC collects all the measured parameters (DC voltages $V_{S M G i}$ and $\mathrm{AC}$ frequency $\left.f_{S M G}\right)$ at the defined sampling interval through the communication link (Fig. 4(a)). Once normalised as in (6), these parameters are sent to the other ILCs. The resulting normalized indices $\left(\dot{V}_{S M G 1}, \dot{V}_{S M G 2}, \ldots \dot{V}_{S M G N}\right.$, where $\mathrm{N}$ represents the number of the participating DC SMGs) are given the symbol $\dot{V}_{S M G i}$ where $i$ stands for the $i^{\text {th }}$ DC SMG and represent the normalised voltages, while $f_{S M G}$ represents the normalised frequency. Then, the collected variables are averaged and the control signal is sent out to the primary control loop of each ILC. This can mathematically be expressed as follows:

$$
\bar{y}_{S M G}=\frac{\sum_{i=1}^{N} V_{S M G_{i}}^{\prime}+f_{S M G}^{\prime}}{N+1}
$$

where $\bar{y}_{S M G}$ is the averaged variable of the $\dot{V}_{S M G i}$ (normalised voltage) and $f_{S M G}$ (normalised frequency) and range between 0 to 1 . The averaged variable $\bar{y}_{S M G}$ is then equalized with each DC SMG's normalised factor $V_{S M G i}$ through the continous feed of the per unit (p.u) error $\left(\gamma_{i}\right)$ to a PI controller. The controller accordingly triggers the power transfer and minimises the difference between $\bar{y}_{S M G}$ and $\dot{V}_{S M G i}$ as in (9). The output value $\left(P_{r e f, i}\right)$ represents the p.u transferred active power reference. This reference triggers the ILC to share the active power proportionally to its rating where the sign of the subtraction process given in (9) gestures the direction of the power flow. The active power reference $\left(P_{r e f, i}\right)$ can be obtained as:

$$
P_{r e f, i}=k_{P}\left(\gamma_{i}\right)+k_{I} \int\left(\gamma_{i}\right) ; \quad \gamma_{i}=\left(\bar{y}_{S M G}-V_{S M G_{i}}^{\prime}\right)
$$

where $k_{P}$ and $k_{I}$ represent the PI control parameters of the outer loop of the ILC. Once the reference power $\left(P_{r e f, i}\right)$ is found through (9), it can then be converted to the active reference current $\left(I^{*}{ }_{i, d}\right)$ according to (10).

$$
I_{i, d}^{*}=\frac{2 \times P_{r e f, i}}{3 \times\left|V_{a b c}\right|}
$$

Here, $\left|V_{a b c}\right|$ represent the AC voltage magnitude of AC SMG. The active current reference $\left(I^{*}{ }_{i, d}\right)$ is then supplied to the inner control loop which has a current and voltage control loop (Fig. 4(b)). These control loops are used in conjunction with the modified vector decoupling control technique which is implemented by feed-forwarding the line voltage components. This not only facilitates the decoupling of the active and reactive power control but also allows operation at a unity power factor when the reference value of the reactive reference current $\left(I_{i, q}^{*}\right)$ is set to zero. The final step in the control process is to transform the $d q$ components of the reference voltage into its corresponding $a b c$ components. The

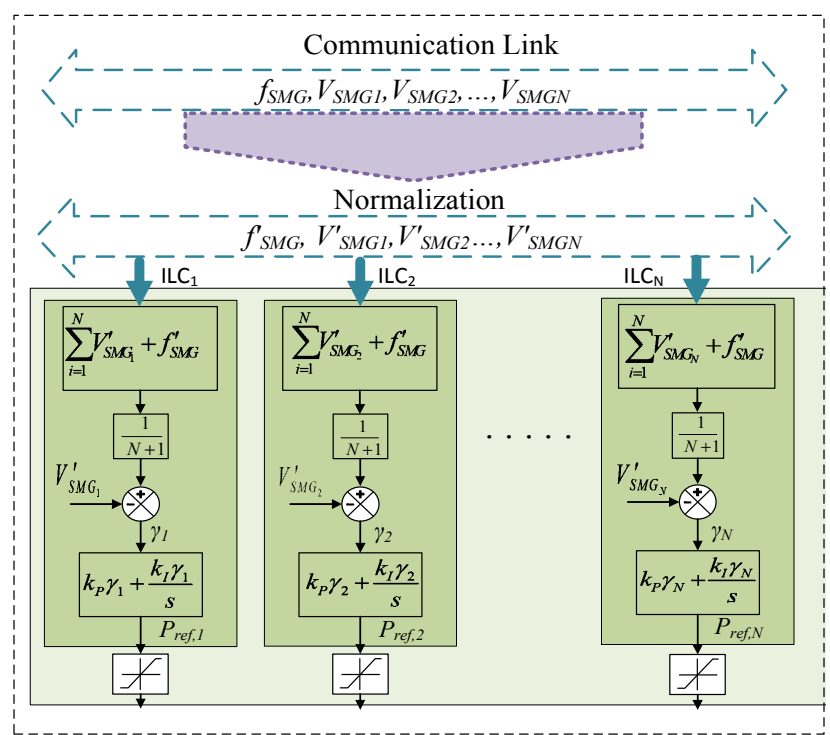

(a)

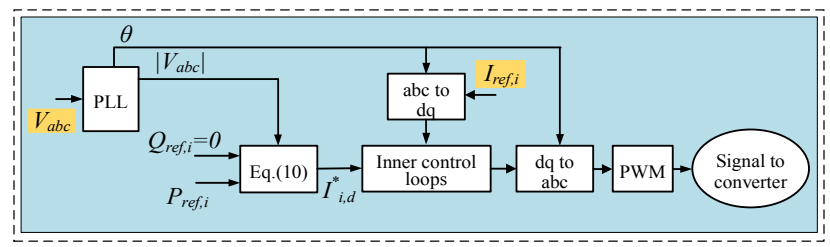

(b)

Figure 4. The proposed control approach diagram

proposed control scheme is totally distributed control system hence, and every ILC has its own local power controller that can produce appropriate control reference for the primary control level by using the measurements of other ILCs in each sample time. While the averaged control scheme in (8) is centralized for simplification purpose, it can be readily adapted to a distributed one by using only neighbouring information.

When all the SMGs are online, and the HMG is considered under normal operating conditions, the proposed approach can lead to a similar output to the conventional one. However, if the AC SMG is disconnected from the system, the transferred power in the conventional method will no more take place even when the remaining SMGs are still connected to the HMG. This is due to the loss of the normalized frequency $\left(f_{S M G}\right)$ that mainly balances the normalized voltages of the DC SMGs $\left(\dot{V}_{S M G s}\right)$ to trigger the ILCs. On the other hand, through the proposed method, the power transfer will continue among SMGs that are connected to the AC common bus even if the $\mathrm{AC} \mathrm{SMG}$ is disconnected from the system.

Moreover, under the proposed scheme and in the case of the AC SMG disconnection mentioned earlier, the voltage and frequency at the $\mathrm{AC}$ common bus are now regulated by one of the ILCs to fill in the absent role of the disconnected AC SMG. The other ILCs continue to manage the power flow as intended.

\section{CASE StUdies AND Discussions}

For verifying the performance and validity of the proposed control approach and power management strategy, a 

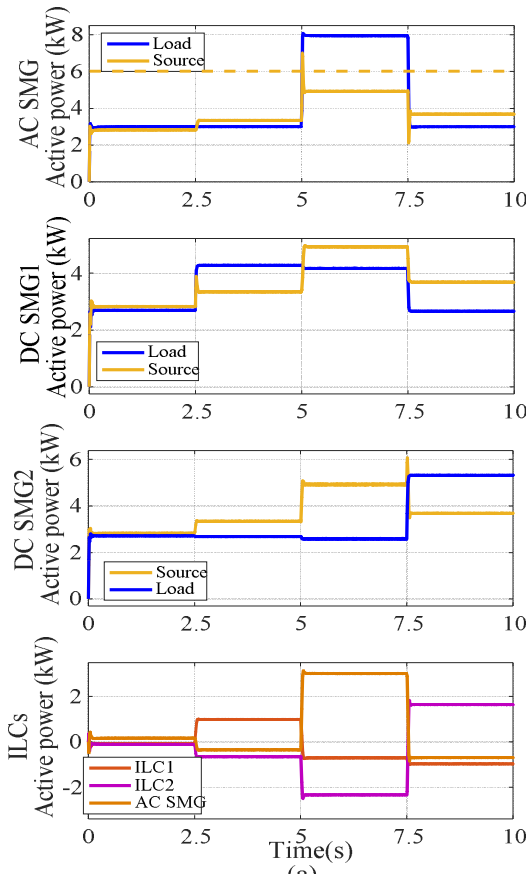

(a)
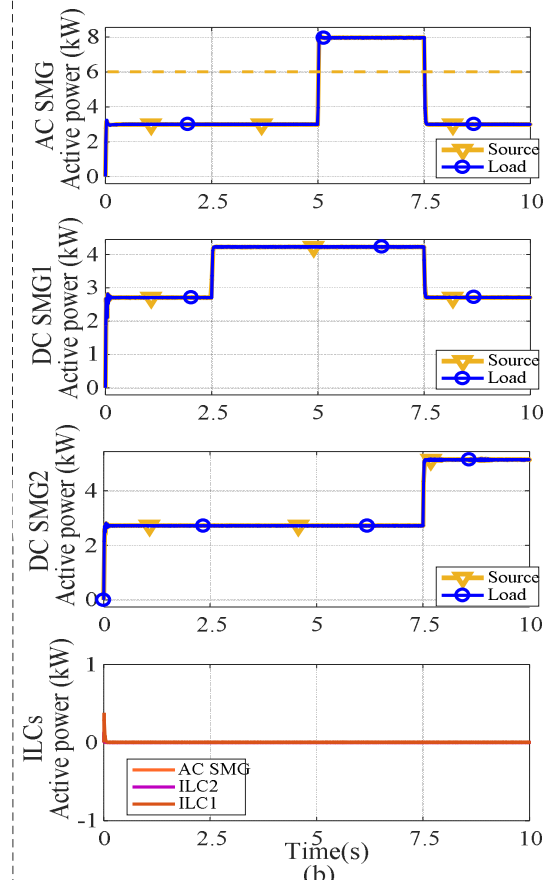
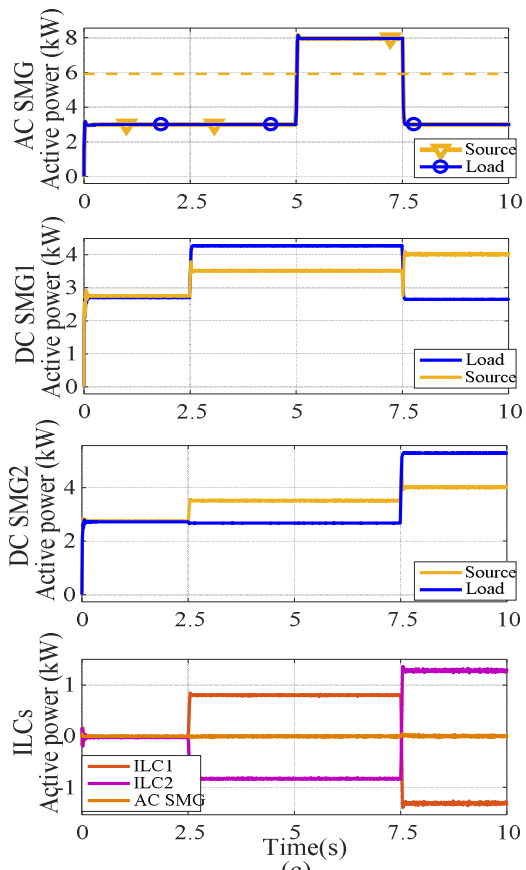

(c)

Figure 5. Simulated power waveforms within hybrid MG for four load step scenarios during three case study (a) case A, (b) case B, (c) case C.

Matlab/Simulink simulation is executed with the presented multi-SMGs structure shown in Fig. 1. DC SMG1 consists of two DGs that is represented by a DC/DC converter. A threephase DC/AC converter is used to represent the AC SMG. Each of the DC SMG2 and AC SMG is supplied through one DG only. The total output power rating of all SMGs is set at the same rating of $6 \mathrm{~kW}$ so that their droop factors $\left(\alpha_{S}, \lambda_{S}\right)$ given through equation (5) are constantly tuned to maintain the generated output powers in a balanced state for different loads. The DC voltage range is $600<V<650$ for DC SMG1 and $400<V<450$ for DC SMG2 while the frequency range for AC $\mathrm{SMG}$ is $49<f<51$. The proposed hybrid microgrid is tested by carrying out a simulation at different loading conditions in each SMG. Such conditions ensure the validity of the findings in terms of the bidirectional power flow. Three case studies are presented to confirm the performance of the proposed approach and make a comparison to the conventional method.

\section{A. Case A: Power Exchange under Normal Conditions Using the Proposed Control Strategy}

In this case, the three SMGs are online, and they are linked to the common AC bus through the ILCs. Power exchange takes place according to the loading status of each SMG. The waveforms of the active power flow for loads, sources, and ILCs are shown in Fig. 5(a). Prior to $t=2.5 \mathrm{sec}$, the SMGs are equally loaded and limited power exchange is noted. The first load transient takes place for the DC SMG1 at $t=2.5 \mathrm{sec}$ at which the load demand increases to $4.3 \mathrm{~kW}$. This triggers the ILCs to transfer active power through the AC common bus to the DC SMG1. In this case, the transferred power is supplied from the AC SMG and DC SMG2 as shown in the ILCs waveform representation in Fig. 5(a). Moving forward, it can be seen that the active power transfer from DC SMG1 and DC SMG2 to the AC SMG due to the load increase at $t=5 \mathrm{sec}$ for the AC SMG. The last load transient takes place at $t=7.5 \mathrm{sec}$ at which DC SMG2 has an added load while DC SMG1 and the AC SMG has considerable load reductions.

\section{B. Case B: Power Exchange when AC SMG is Isolated Using Conventional Control Method}

Meanwhile, the AC SMG may need to detach from the HMG during an emergency or planned maintenance. Fig. 5(b) shows the power flow waveforms for all the SMGs. It can be noted that for each SMG, the power demand is identical to that of the generated power. Accordingly, there is no power transferred among the SMGs as each SMG is capable of meeting its own demand. Using this method (conventional), load shedding is usually needed to overcome the case in which the load of an SMG exceeds its supply capacity.

\section{Case C: Power Exchange when AC SMG is Isolated Using the Proposed Control Method}

This case study demonstrates the flexible performance of the proposed method as compared to the conventional one. Fig. 5(c) illustrates the continuity of power transfer between
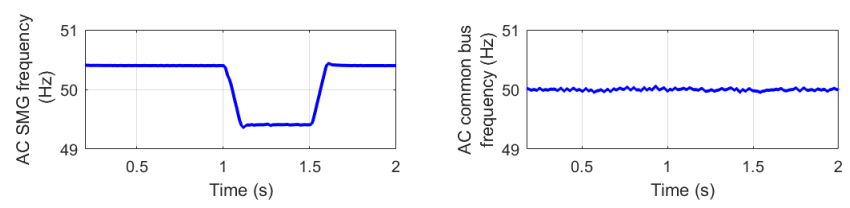

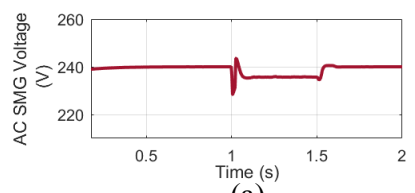

(a)

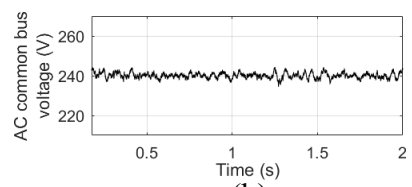

(b)
Figure 6. Comparison between (a) AC SMG and (b) AC common bus for case $\mathrm{C}$. 

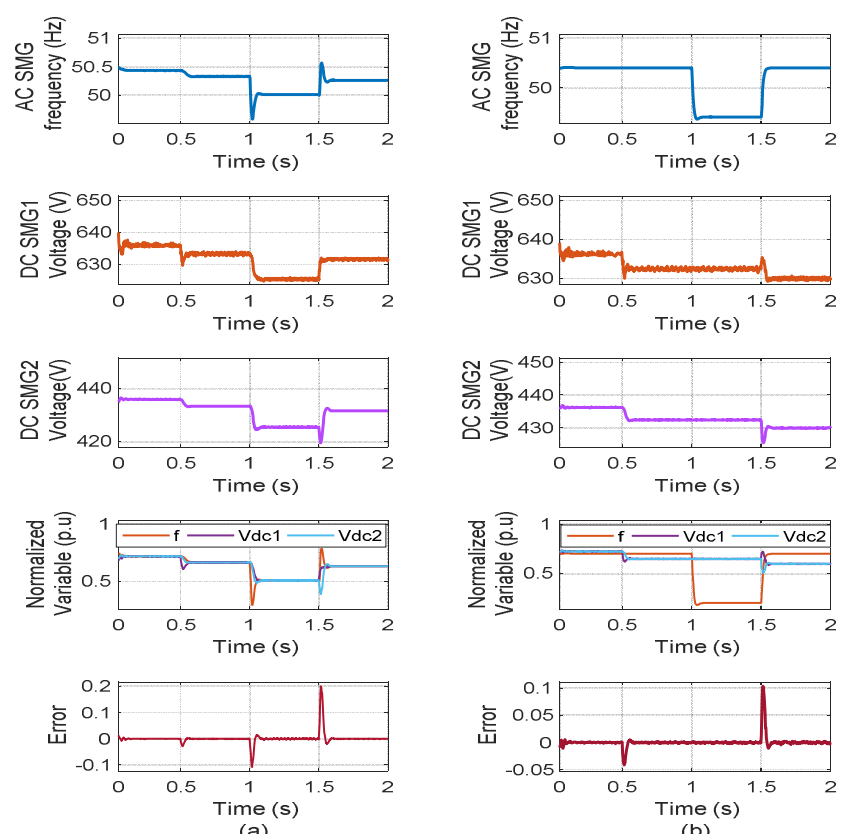

Figure 7. Frequency and voltage waveforms for (a) case A, and (b) case C

DC SMG1 and DC SMG2 when the AC SMG is cut off from the HMG. The figure depicts two load transients that occur at $t=2.5 \mathrm{sec}$ and $t=7.5 \mathrm{sec}$. Unlike the previous case, the DC SMGs are still connected and interchange power despite the loss of the AC SMG as part of the HMG. This is further shown through the DC SMG1 and DC SMG2 waveforms.

Fig. 6 shows the difference in voltage and frequency between the AC SMG (Fig. 6(a)) and the AC common bus (Fig. 6(b)) throughout the case study C. It confirms the continuous operation of the $\mathrm{AC}$ common bus while the $\mathrm{AC}$ SMG is isolated from the HMG. It also can be noted that the frequency and voltage in the AC SMG are regulated by its local controller in accordance with the droop control. On the other hand, the $\mathrm{AC}$ common bus under the proposed method is regulated by one of the DC SMG ILCs with a standard external reference to substitute the AC SMG role. The main aim of such coordination is to maintain the operation of the $\mathrm{AC}$ common bus when the $\mathrm{AC}$ SMG is no longer connected to the HMG. Fig. 7 shows the voltage and frequency waveforms of all SMGs for the case study A (Fig. 7(a)) and case study C (Fig. 7(b)) throughout the four load transients. It also shows the normalized variables of the three SMGs. It clearly shows that in case A, all the variables were equalized since the generated power is balanced for all SMGs. However, in Fig. 7(b), the normalized variables are only equalized for DC SMG1 and DC SMG2 as these SMGs are the ones connected to the HMG. Fig. 7(b) also shows the advantage of the proposed method in terms of sharing active power between DC SMGs when the AC SMG is isolated from the HMG. The voltage and frequency variations are relatively small for each SMG since the linking of the DC SMGs increases the total power rating. Accordingly, the whole system is considerably more stable.

\section{CONCLUSIONS}

In this paper, a new control method for accurate active power sharing for the hybrid AC/DC microgrid with multiple SMGs is presented. The proposed method suggests a distributed control system that ensures global controllability for the ILCs specifically in the case of multiple SMGs. This overcomes the total dependency on a specific variable for stable power exchange which is the case in the conventional method. The proposed control method has been compared with the conventional one under different conditions. The various simulations and its results verify that the proposed scheme ensures a continous and seamless power transfer irrespective of the failure of the AC SMG.

\section{REFERENCES}

[1] F. Blaabjerg, R. Teodorescu, M. Liserre, and A. V. Timbus, "Overview of control and grid synchronization for distributed power generation systems," IEEE Trans. Ind. Electron., vol. 53, pp. 1398-1409, 2006.

[2] F. Nejabatkhah and Y. W. Li, "Overview of power management strategies of hybrid AC/DC microgrid," IEEE Trans. Power Electron., vol. 30, no. 12, pp. 7072-7089, 2015.

[3] M. Abuhilaleh, L. Li, M. Begum, and J. Zhu, "Power management and control strategy for hybrid AC/DC microgrids in autonomous operation mode," in Electrical Machines and Systems (ICEMS), 2017 20th International Conference on, 2017, pp. 1-6: IEEE.

[4] E. Unamuno and J. A. Barrena, "Hybrid ac/dc microgrids-Part II: Review and classification of control strategies," RENEW SUST ENERG $R E V$, vol. 52, pp. 1123-1134, 2015.

[5] J. M. Guerrero, J. C. Vasquez, J. Matas, D. Vicuna, L. García, and M. Castilla, "Hierarchical control of droop-controlled AC and DC microgrids-A general approach toward standardization," IEEE Trans. Ind. Electron., vol. 58, no. 1, pp. 158-172, 2011.

[6] X. Liu, P. Wang, and P. C. Loh, "A Hybrid AC/DC Microgrid and Its Coordination Control," IEEE Trans. Smart Grid, vol. 2, pp. 278-286, 2011.

[7] J. C. Vasquez, J. M. Guerrero, M. Savaghebi, J. Eloy-Garcia, and R. Teodorescu, "Modeling, analysis, and design of stationary-referenceframe droop-controlled parallel three-phase voltage source inverters," IEEE Trans. Ind. Electron., vol. 60, no. 4, pp. 1271-1280, 2013.

[8] N. Eghtedarpour and E. Farjah, "Power control and management in a hybrid AC/DC microgrid," IEEE Trans. Smart Grid, vol. 5, no. 3, pp. 1494-1505, 2014

[9] H. Han, X. Hou, J. Yang, J. Wu, M. Su, and J. M. Guerrero, "Review of power sharing control strategies for islanding operation of $\mathrm{AC}$ microgrids," IEEE Trans. Smart Grid, vol. 7, no. 1, pp. 200-215, 2016.

[10] J. M. Guerrero, M. Chandorkar, T.-L. Lee, and P. C. Loh, "Advanced control architectures for intelligent microgrids-Part I: Decentralized and hierarchical control," IEEE Trans. Ind. Electron., vol. 60, no. 4, pp. 1254-1262, 2013.

[11] M. S. Rahman, M. Hossain, and J. Lu, "Coordinated control of threephase AC and DC type EV-ESSs for efficient hybrid microgrid operations," ENERG CONVERS MANAGE, vol. 122, pp. 488-503, 2016.

[12] M. Nehrir et al., "A review of hybrid renewable/alternative energy systems for electric power generation: Configurations, control, and applications," IEEE Trans. Sustain. Energy, vol. 2, pp. 392-403, 2011.

[13] P. C. Loh, D. Li, Y. K. Chai, and F. Blaabjerg, "Autonomous operation of hybrid microgrid with AC and DC subgrids," IEEE Trans. Power Electron., vol. 28, no. 5, pp. 2214-2223, 2013.

[14] P. Wang, C. Jin, D. Zhu, Y. Tang, P. C. Loh, and F. H. Choo, "Distributed control for autonomous operation of a three-port AC/DC/DS hybrid microgrid," IEEE Trans. Ind. Electron., vol. 62, no. 2, pp. 1279-1290, 2015.

[15] Q. Xu, J. Xiao, P. Wang, and C. Wen, "A Decentralized Control Strategy for Economic Operation of Autonomous AC, DC, and Hybrid AC/DC Microgrids," IEEE Trans. Energy Convers, vol. 32, no. 4, pp. 1345-1355, 2017. 